\title{
Cardiovascular effects of epinephrine and dipivalyl epinephrine applied topically to the eye in patients with glaucoma
}

\author{
CHARLES R. KERR, IAN HASS, STEPHEN M. DRANCE, MAX B. WALTERS, AND \\ MICHAEL SCHULZER
}

From the Division of Cardiology, Department of Medicine, and the Department of Ophthalmologv, University of British Columbia and the Vancouver General Hospital, Vancouver, BC, Canada

\begin{abstract}
SUMmARY The cardiovascular effects of topical ophthalmological preparations of $2 \%$ epinephrine (EPI), $0 \cdot 1 \%$ dipivalyl epinephrine (DPE), and placebo were studied in double-blind fashion in 20 patients with glaucoma. Both drugs and placebo produced a decrease in heart rate $(2 \pm 3$ beats $/ \mathrm{min})$ which, although small, was highly significant $(\mathrm{p}<0 \cdot 001)$. Neither drug nor placebo produced a significant effect on mean or systolic blood pressure (BP) over the group as a whole $(\mathrm{p}>0 \cdot 1)$. However, 5 of 20 patients responded to EPI with a significant rise in mean or systolic BP (defined as a change greater than mean change $\pm 2 \mathrm{SD}$ ), whereas there were no such responses to DPE or placebo. One patient developed marked ventricular ectopy after EPI. We conclude that EPI may cause cardiovascular side effects in a high percentage of patients (25\% in this study) through individual susceptibility rather than a predictable effect. DPE, a new epinephrine analogue. appears to be devoid of these effects.
\end{abstract}

Topical ophthalmic preparations are commonly employed in the treatment of patients with glaucoma. By the nature of this condition a large number of such patients fall into an older age group, a group also with a high prevalence of heart disease.

Little has been reported on the cardiovascular effects of topical eye drops. Such reports have been largely case studies, and as yet no prospective trial has been undertaken to analyse the effects of these preparations. The purpose of this study was to evaluate in a prospective, randomised, double-blind fashion the cardiovascular response to topically applied epinephrine (EPI), dipivalyl epinephrine (DPE, a new epinephrine analogue), and placebo (P) in patients with chronic open-angle glaucoma.

\section{Patients and methods}

Twenty consecutive patients with chronic open-angle glaucoma were selected as they returned for followup care to the Department of Ophthalmology, University of British Columbia. All gave informed,

Correspondence to Dr S M Drance. Department of Ophthalmology, 2577 Willow Street, Vancouver. BC. Canada V5Z 3N9. written consent. Each patient was assessed by history, physical examination, and electrocardiogram (ECG) and assigned to one of 2 groups, one with and one without heart disease.

Each patient underwent a trial of 3 different topical ophthalmic preparations on 3 separate morningsMonday, Wednesday, and Friday. All eye drops were stopped at least 96 hours before the trial, and EPI was stopped one week prior to the trial with only oral acetazolamide being permitted to be continued. These 3 medications were supplied in coded form by Allergan Pharmaceutical Inc. with each patient's drugs individually coded and administered in randomised order to each patient. The trial was thus double-blind. The medications studied were: (1) $2 \%$ epinephrine (EPI), (2) $0.1 \%$ dipivalyl epinephrine (DPE), and (3) placebo (P). Each was administered by a physician as a single drop to each eye.

The experiments were carried out in the fasting state with patients in the sitting position. Heart rate (HR) and rhythm were assessed by a Holter ambulatory monitoring system, recording for one hour prior to administration of eye drops and for one hour following. ECGs were recorded prior to administration and 5 and 10 minutes following in order to assess 
ST-T changes. Blood pressure (BP) was recorded by sphygmomanometer in duplicate before and 1, 3,5, and 30 minutes after medication. Blood pressure was taken by the same observer in all cases. Mean BP was calculated by the formula:

$$
\text { Mean } \mathrm{BP}=\frac{2 \times \text { diastolic }+1 \times \text { systolic }}{3}
$$

Intraocular pressure (IOP) was recorded before and 30.60 , and 120 minutes after drugs by a single observer using the Goldmann applanation tonometer. Patients were asked to report any symptoms, either systemic or local.

Each 2-hour Holter recording was analysed by Avionics Dynamic Electrocardioscanner (Model $660 \mathrm{~A})$. The mean HR and total number of ventricular ectopic beats (VEBs) were calculated for the 30minute period after instituting monitoring was used to acclimatise the patients to the procedure, and the HR and VEBs counted during this period were not used for analysis.

Mean and systolic BP were analysed by comparing the values before medication (mean of 2 readings) to the mean of 2 readings taken at 3 and 5 minutes after medication. Similarly IOP was compared before and 30,60 , and 120 minutes after drug administration.

ECGs were read without knowledge of the patients by 2 members of the Division of Cardiology, and discrepancies in interpretation were settled by a third reader. Changes in ST-T segments were specifically noted.

Statistical analysis of the cardiovascular data was performed by analysis of variance nested model, with which comparisons were made between each drug in each patient group and in the 20 patients as a whole. Ophthalmological data was studied by analysis of variance of the effects of each drug at 30,60 , and 120 minutes. In analysing the IOP data one eye was randomly selected from each patient and analysis done on the IOP changes in that eye. An unpaired Student's $t$ test was used to compare the ages of the patients in the 2 groups. Student's paired $t$ test was used to compare the heart rate before and after drug administration in all 60 drug trials.

\section{Results}

Nine of the 20 patients were classified as having no heart disease (group A), whereas 11 were classified as having heart disease (group B). Group A was composed of 6 males and 3 females, with a mean age of $66 \pm 13(\mathrm{SD})$. Group B had 5 males and 6 females. and their mean age of $74 \pm 9$ was not significantly different from that of group $A(p>0 \cdot 05)$.

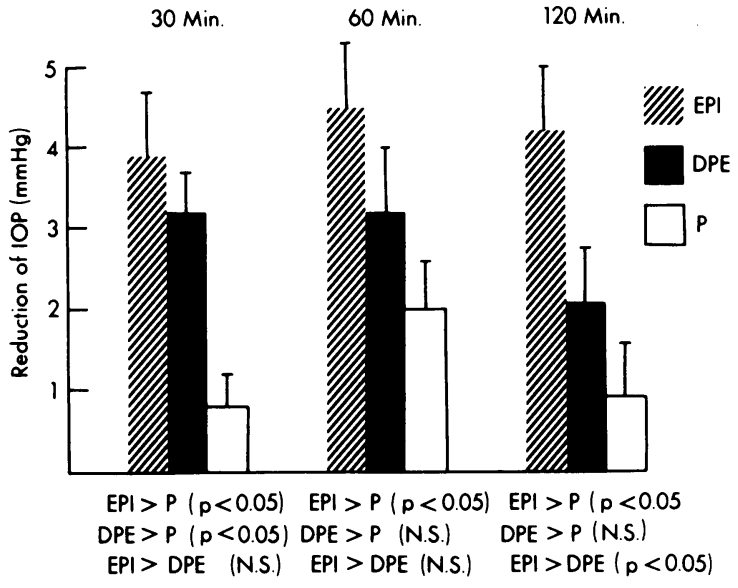

Fig. 1 The reduction in intraocular pressure (IOP) observed after the administration of epinephrine (EPI). dipivalyl epinephrine (DPE), and placebo $(P)$. The variations shown represent standard error of the mean.

Of the 11 patients in group $B 3$ had heart disease on clinical grounds (aortic stenosis 2, raised jugular venous pressure 1). Nine had abnormal ECGs (old myocardial infarction 3, intraventricular conduction delay 4 , left ventricular hypertrophy 3 , and ST-T wave abnormalities 4 ). Fourteen of 20 patients had received EPI in the past, and 13 of the 14 had stopped the drug owing to local side effects.

All 20 patients successfully completed the study.

OPHTHALMOLOGICAL DATA

The effects of the 3 treatments on IOP was analysed in the entire 20 patients as a single block. The results comparing IOP before medication and 30,60), and 120 minutes after medication are shown in Fig. 1.

At 30 minutes the reduction in IOP induced by EPI $(3.9 \pm 3.4 \mathrm{mmHg})$ was equal to that of DPE $(3.2 \pm$ $2 \cdot 1 \mathrm{mmHg}$ ), and these were both statistically greater $(p<0.05)$ than the reduction seen with $P(0.8 \pm$ $1.9 \mathrm{mmHg}$ ). At 60 minutes EPI reduced IOP by $4.5 \pm$ $3.7 \mathrm{mmHg}$, which was significantly greater $(\mathrm{p}<0.05)$ than the effect of $P(2 \cdot 0 \pm 3.5 \mathrm{mmHg})$ but not different $(p>0.05)$ from DPE $(3.2 \pm \mathrm{mmHg})$. While the effect of DPE at 60 minutes was greater than $P$, this difference was not significant $(p>0 \cdot 05)$.

\section{CARDIOVASCULAR DATA}

Neither of the 2 medications nor placebo produced a significant change in either systolic or mean BP. The response to the preparations did not differ between the groups with or without heart disease, nor was there any difference among the 3 preparations $(p>0 \cdot 1)$. These responses are shown in Fig. 2, where the changes in systolic and mean BP are shown for 
SYSTOLIC BP

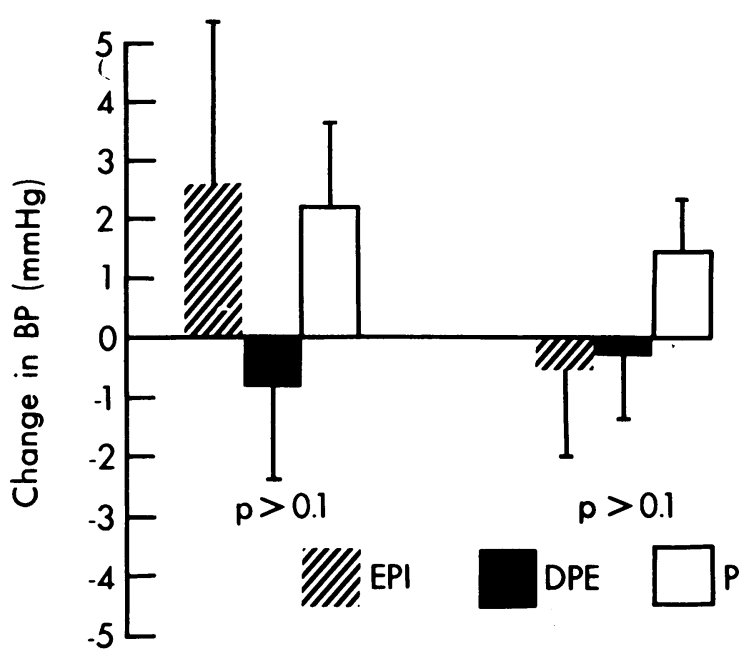

Fig. 2 The changes in mean and systolic blood pressure $(B P)$ seen after the instillation of epinephrine (EPI). dipivalvl epinephrine (DPE. and placebo $(P)$. Variations shown represent standard error of the mean.

each drug in the group of 20 patients considered as a whole. The mean changes and standard deviations in blood pressure for all 60 patient drug trials were $1.4 \pm$ $8.5 \mathrm{mmHg}$ for systolic BP and $0.2 \pm 5.3 \mathrm{mmHg}$ for mean BP.

Significant changes in individuals were then sought by identifying any change in systolic or mean BP greater than 2 standard deviations outside the mean change of BP. With these criteria a significant change was thus defined as a change of systolic BP of $19 \mathrm{mmHg}$ or greater or a change of mean BP of $11 \mathrm{mmHg}$ or greater. Four patients showed a significant change in systolic BP of $19,21,21$, and $34 \mathrm{mmHg}$ respectively. All these increases followed EPI administration; 2 were in group $A$ and 2 in group $B$, the ages of the patients being $56,74,83$, and 79. No changes in systolic BP of this magnitude were seen
Table 1 Significant changes in blood pressure

\begin{tabular}{|c|c|c|c|c|c|c|}
\hline \multirow[t]{2}{*}{ Patient } & \multicolumn{3}{|c|}{$\triangle B P$ Svstolic $(\mathrm{mmHg})$} & \multicolumn{3}{|c|}{$\triangle B P$ Mean $(m m H g)$} \\
\hline & $E P I$ & $D P E$ & $P$ & $E P I$ & $D P E$ & $P$ \\
\hline $1 \mathrm{~A}$ & $19^{*}$ & 4 & 0 & $11^{*}$ & 6 & 1 \\
\hline $5 \mathrm{~A}$ & $21^{*}$ & 3 & 12 & 5 & 6 & 4 \\
\hline $8 A$ & 17 & -2 & 6 & $12^{*}$ & 3 & 3 \\
\hline IB & $34^{*}$ & $\overline{5}$ & -4 & -2 & 0 & -4 \\
\hline $10 \mathrm{~B}$ & $21^{*}$ & 8 & 2 & 6 & 4 & 9 \\
\hline
\end{tabular}

*Denotes changes in blood pressure (BP) considered to be significant (mean $\mathrm{B} \mathrm{BP} \pm \mathrm{SD}$ ).

in any patient after DPE or $\mathrm{P}$, and no major drop in systolic BP occurred. One of these 4 patients also showed a significant rise in mean BP $(11 \mathrm{mmHg})$ after EPI, while one additional 56-year-old patient in group A showed an increase in mean BP of $12 \mathrm{mmHg}$. again after EPI. This individual had a rise in systolic BP of $17 \mathrm{mmHg}$. just below the level of significance in Table 1. Four of these 5 patients had previously received EPI, whereas one had never received the drug.

Mean HR showed a significant decrease regardless of the agent used. In all 60 drug administrations HR fell $2 \pm 3$ beats/minute from $68 \pm 11$ to $66 \pm 10$ beats/minute. While this decrease is small, it was very consistent and thus reached a high degree of significance $(p<0.001)$. There were no differences among the 2 medications and placebo in their effect on HR ( $p>0 \cdot 05)$.

Two patients in group $A$ and 4 in group $B$ had more than one VEB on any of their recordings. Table 2 shows the total number of VEBs from the 30-minute period before drug administration and the 30-minute period following. The numbers were too small and variable for statistical analysis, but changes in the number of VEBs appear to be important, and one patient is discussed below.

This patient was a 79-year-old male who responded dramatically to the instillation of EPI. With P and DPE he showed no change in number of VEBs per 30 minute period ( 6 to 2 and 1 to 1 respectively). Prior to

Table 2 Ventricular ectopic beats (VEBs) before and after topical medication

\begin{tabular}{|c|c|c|c|c|c|c|}
\hline \multirow[t]{2}{*}{ Patient } & \multicolumn{2}{|l|}{ Epinephrine } & \multicolumn{2}{|l|}{$D P E$} & \multicolumn{2}{|l|}{ Placebo } \\
\hline & $\begin{array}{l}V E B s / 30 \mathrm{~min} \\
\text { Before }\end{array}$ & $\begin{array}{l}V E B s / 30 \mathrm{~min} \\
\text { After }\end{array}$ & $\begin{array}{l}V E B s / 30 \mathrm{~min} \\
\text { Before }\end{array}$ & $\begin{array}{l}V E B s / 30 \mathrm{~min} \\
\text { After }\end{array}$ & $\begin{array}{l}V E B s / 30 \mathrm{~min} \\
\text { Before }\end{array}$ & $\begin{array}{l}V E B s / 30 \mathrm{~min} \\
\text { After }\end{array}$ \\
\hline $5 \mathrm{~A}$ & 35 & 31 & 28 & 34 & 28 & 16 \\
\hline $6 A$ & 8 & 11 & 6 & 9 & 2 & 5 \\
\hline $1 \mathrm{~B}^{*}$ & 2 & 362 & 1 & 1 & $\overline{6}$ & 2 \\
\hline $4 B$ & $\overrightarrow{1}$ & 6 & 8 & 2 & 6 & 8 \\
\hline $5 B$ & 14 & 5 & 305 & $40 \overline{7}$ & 167 & 142 \\
\hline $7 \mathrm{~B}$ & 36 & 16 & 33 & 14 & 39 & 31 \\
\hline
\end{tabular}

*Patient with a major response following epinephrine: see discussion in text and Fig. 3. 




Fig. 3 The time course of ventricular ectopic beats (VEBs) before and following the instillation of $2 \%$ epinephrine (EPI) in patient $1 B$ (see Table 2 and text for discussion). The time of instillation of EPI is indicated by the arrow.

EPI his Holter recording showed multiple atrial ectopic beats but only 2 VEBs per 30 minutes. Following EPI he had 362 VEBs in the first 30 minutes. beginning with 1 minute of drug administration and returning to baseline within 60 minutes (Fig. 3). His ECGs are shown in Fig. 4. This same individual also had a large increase in systolic BP (158 to $194 \mathrm{mmHg})$ after EPI but no change after DPE and P (patient $1 B$ in Table 1). In spite of these changes he remained asymptomatic.

The reading of successive ECGs by 2 observers showed no significant change in wave form with any medication. Specifically there were no changes in ST-T waves suggesting increasing ischaemia.

No patient reported any svmptoms referrable to the cardiovascular system during these studies.

\section{Discussion}

The efficacy of EPI in lowering IOP in patients with chronic open-angle glaucoma has been well established ${ }^{1-4}$ and is thought to be due to the adrenergic effect on the ciliary body and the outflow pathways. ${ }^{3-5}$ However, a significant number of patients on this medication for a long time develop localised side effects requiring discontinuation of the drug. These side effects include chronic irritation and hyperaemia. tearing, persistent headache, blurring of vision, and corneal oedema. ${ }^{4}$ Hypersensitivity, blepharoconjunctivitis, ${ }^{4}$ macular oedema in the aphakic eye ${ }^{6}$ and black corneal deposits ${ }^{48}$ have been described. Becker and Morton ${ }^{4}$ report that only $20 \%$ of patients could continue EPI for 4 to 5 years owing to the incidence and severity of these local effects.

Several reports have described systemic cardiovascular reactions to EPI. Weiner and Alvis' noted palpitations, tremor, sweating, and rising $\mathrm{BP}$ in



Fig. 4 Electrocardiograms of patient 1 B taken (A) before instillation of 2\% Epinephrine (EPI) and (B) 5 minutes following the instillation of $2 \%$ EPI. See Table 1, Fig. 3, and text for discussion. 
patients receiving both subconjunctival and topical epinephrine compounds. Becker and Morton ${ }^{4}$ described palpitations in 4 of 50 patients receiving $2 \%$ epinephrine hydrochloride of $2 \%$ epinephrine bitartrate. Ballin' et al. showed that patients receiving EPI for a long time had more VEBs over an 8-minute period of tonography than did those receiving other medication. Becker ${ }^{10}$ et al. reported at least one VEB per 4-minute period of tonography in $69 \%$ of patients receiving EPI for primary open-angle glaucoma compared with $19 \%$ of patients receiving EPI for secondary glaucoma. Both of these reports show a high incidence. of isolated VEBs but do not demonstrate their occurrence in a temporal relationship to EPI administration.

Lansche $^{11}$ reported 2 cases in which 2 drops of $2 \%$ EPI resulted in transient hypertension and tachycardia lasting about 15 minutes. ECGs were not recorded during these episodes. Both cases were associated with recent incision of a chalazion or corneal epithelial disruption.

Two drops $(0.05 \mathrm{ml} / \mathrm{drop})$ of $2 \%$ EPI contain $2 \cdot 0 \mathrm{mg}$ of epinephrine. The usual recommended subcutaneous dose in patients with allergic reactions or asthma is 0.1 to $0.3 \mathrm{ml}$ of $1: 1000$ solution $(0.1$ to $0.3 \mathrm{mg}$ ). Thus the doses in ophthalmological preparations are considerably higher and, if readily delivered into the circulation, might produce significant systemic effect.

DPE is dipivalyl ester of epinephrine, which, because of greater lipophilic properties, has increased absorption into the eye. ${ }^{12}{ }^{13}$ Once inside the eye DPE is hydrolysed to form EPI.$^{13}$ It has been estimated that DPE may reach concentrations in the eye about 10 times that of an equal dose of EPI without reaching significant systemic levels. ${ }^{13}$ Thus it is possible to use smaller doses of DPE ( $(0 \cdot 1 \%$ solution) than EPI ( $2 \%$ solution) to obtain a similar effect. This theoretically should reduce the incidence of systemic cardiovascular side effects. DPE has been shown to lower the intraocular pressure (IOP) over a one-month period when given in a dose of 1 drop of $0 \cdot 1 \%$ solution twice daily. ${ }^{12}$

The present study confirms the efficacy of both DPE and EPI in acutely lowering the IOP after a single administration. It would appear from our data that both have an equal effect initially, but after 60 and 120 minutes EPI is more effective in lowering IOP. It would be inappropriate to extrapolate these findings to long-term effects of these drugs. It is worth noting again that the amount of DPE used is $5 \%$ that of EPI on a purely quantitative comparison.

From a cardiovascular perspective this study has shown that neither DPE nor EPI had any statistically significant effects on BP or on the incidence of VEBs or ischaemic ECG changes. Furthermore there was no statistical difference betweeen the 2 drugs in these effects in patients with or without heart disease.

The response of the heart rate of the 2 medications as well as placebo was too slow. While this slowing was slight, it was extremely reproducible and thus attained a high degree of significance. The HR was allowed to stabilise for a minimum of 30 minutes before the baseline was recorded. and therefore a natural slowing due to progressive patient relaxation is not likely. The mechanism of this action is not clear. The fall in HR was not associated with a rise in BP. and thus a reflex bradycardia cannot be implicated. It is possible that corneal irritation as a result of local anaesthesia, tonometry, and drug administration may result in vagal discharge and thus a decrease in the rate of sinus node activity.

There was no consistent effect on either systolic or mean BP with EPI, DPE. or P. While the change in BP with EPI was more than with DPE. this was not statistically different. However, after EPI 5 patients did respond with increases in systolic and/or mean BP which are considered individually significant. These occurred only with EPI. and such increases were not seen in any patients after DPE or P. This would suggest that, while EPI does not have a predictable effect on BP, it may result in a significant increase in BP with a reasonably high frequency $(25 \%$ in our study) in susceptible individuals. Three of these 5 patients had a significant increase in systolic BP but insignificant changes in mean BP. This is due to a fall in diastolic blood pressure, which. due to the weighting of diastolic pressure in the calculation of mean $\mathrm{BP}$, may counterbalance the rise in systolic BP. This would explain in Fig. 2 why, although the changes in BP are not statistically significant, systolic pressure appears to increase and mean BP to decrease after EPI. These variable effects on BP may be partly explained by the mixed $\alpha$ and $\beta$ effects of epinephrine.

Six patients had ventricular ectopy prior to instillation of drops, and as a group they showed no consistent change with medication. However, one showed a dramatic increase in the incidence of ventricular ectopic beats after EPI (as well as a major rise in BP), again suggesting a susceptibility to EPI in certain individuals. This susceptibility may represent a greater systemic absorption of the drug from the eve or it may represent an increased sensitivity to circulating epinephrine. It is not possible to differentiate these mechanisms from this study.

It is of interest that DPE showed no evidence of cardiovascular systemic reactions other than the minor decrease in HR previously described. This is in contrast to the $25 \%$ incidence of cardiovascular effects following EPI. This would support the theoretical advantage of DPE over EPI in that much lower concentrations of DPE $(0 \cdot 1 \%)$ than EPI 
$(2.0 \%)$ may be used to obtain an advantageous effect on IOP. As a consequence less catecholamine is absorbed, and thus the chance of systemic reaction is decreased. The cardiovascular side effects occurred apparently equally in the groups with and without overt heart disease, suggesting a need for caution in any patient in this older age group who might well have undiagnosable cardiovascular disease.

Several conclusions may be drawn from this study. Firstly, all topical agents in this study produced a small decrease in the heart rate. probably as a result of a nonspecific effect. possibly vagally mediated. Secondly. epinephrine may produce significant cardiovascular side effects in certain susceptible individuals, although there appears to be no uniformly predictable response. This supports previous case reports and again reasserts the need for caution in the use of topical epinephrine. Thirdly. DPE appears to be effective in lowering IOP on a short-term basis and appears to be completely devoid of systemic cardiovascular side effects. In the doses studied EPI appears to be more effective in lowering IOP than DPE after 60 minutes, possibly because of the much lower dose of DPE.

This work was supported in part by Allergan Pharmaceutical Inc.. Irvine. California.

I.H. was in receipt of a Bausch and Lomb fellowship.

\section{References}

1 Weiner M. Alvis BY. The use of concentrated epinephitine preparations in glaucoma. ititis. and related conditions. Am J Ophthalmol 1937: 20: 497-504.

2 Garner LL. Johnstone WW. Ballintine EJ. et al. Effect of 2\% levo-rotary epinephrine on the intraocular pressure of the glaucomatous eve. Arch Ophthalmol 1959: 62: 230-8.

3 Becker B. Pettit TH. Gay AJ. Topical epinephrine therapy of open-angle glaucoma. Arch Ophthalmol 1961: 66: 219-25.

4 Becker B. Morton WR. Topical epinephrine in glaucoma suspects. Am J Ophthalmol 1966: 62: 272-7.

5 Sears ML. The mechanism of action of adrenergic drugs in glaucoma. Invest Ophthalmol Visual Sci 1966: 5: 115-9.

6 Nackool RJ. Muldoon T. Fortier A. et al. Epinephrine-induced cystoid macular edema in aphakic eves. Arch Ophthalmol 1977: 95: 791-3.

7 Ferry AP. Zimmerman LE. Black cornea: a complication of topical use of epinephrine. Am J Ophthalmol 1964: 58: 205.

8 Corwin ME. Spencer WH. Conjunctival melanin depositions. Arch Ophthalmol 1963: 69: 317-21.

9 Ballin N. Becker B. Goldman ML. Svstematic effects of epinephrine applied topically to the eve. Invest OphthalmolVisual Sci. 1966: 5: 125-9.

10 Becker B. Montgomery SW. Kass MA. et al. Increased ocular and svstemic responsives to epinephrine in primary open-angle glaucoma. Arch Ophthalmol 1977: 95: 789-90.

11 Lansche RK. Svstemic reactions to topical epinephrine and phenylephrine. Am J Ophthalmol 1966: 62: 95-98.

12 Kaback MB. Podos SM. Harbin TS. et al. The effects of dipivalyl epinephrine on the eve. Am J Ophthalmol 1976; 81: 768-72.

13 Wei C. Anderson JA. Leopold I. Ocular absorption and metabolism of topically applied epinephrine and dipivalyl ester of epinephrine. Invest Ophthalmol Visual Sci. 1978: 17: 315-21. 